\title{
Rapid identification of red-flesh loquat cultivars using EST-SSR markers based on manual cultivar identification diagram strategy
}

\author{
X.Y. Li, H.X. Xu and J.W. Chen \\ Institute of Horticulture, Zhejiang Academy of Agricultural Sciences, \\ Hangzhou, Zhejiang, China \\ Corresponding author: J.W. Chen \\ E-mail: chenjunwei@mail.zaas.ac.cn
}

Genet. Mol. Res. 13 (2): 3384-3394 (2014)

Received December 6, 2012

Accepted May 15, 2013

Published April 29, 2014

DOI http://dx.doi.org/10.4238/2014.April.29.17

\begin{abstract}
Manual cultivar identification diagram is a new strategy for plant cultivar identification based on DNA markers, providing information to efficiently separate cultivars. We tested 25 pairs of apple EST-SSR primers for amplification of PCR products from loquat cultivars. These EST-SSR primers provided clear amplification products from the loquat cultivars, with a relatively high transferability rate of $84 \%$ to loquat; 11 pairs of primers amplified polymorphic products. After analysis of 24 red-fleshed loquat accessions, we found that only 7 pairs of primers could clearly separate all of them. A cultivar identification diagram of the 24 cultivars was constructed using polymorphic bands from the DNA fingerprints and EST-SSR primers. Any two of the 24 cultivars could be rapidly separated from each other, according to the polymorphic bands from the cultivars; the corresponding primers were marked in the correct position on the cultivar identification diagram. This red-flesh loquat cultivar identification diagram can separate the 24
\end{abstract}


red-flesh loquat cultivars, which is of benefit for loquat cultivar identification for germplasm management and breeding programs.

Key words: Loquat; EST-SSR; Marker; Polymorphic; Manual cultivar identification diagram

\section{INTRODUCTION}

Loquat (Eriobotrya japonica Lindl.) is native to the subtropical area of China, and it is an evergreen fruit tree that blooms in fall and early winter. The species belongs to the subfamily Maloideae of Rosaceae. In spite of the specific epithet japonica, it originated in China and has become an alternative to the main fruit crops (Soriano et al., 2005; Gisbert et al., 2009). On the basis of fruit traits, loquat can be sorted into red- and white-flesh cultivars (Fu et al., 2012). Loquat fruits contain a host of active phytochemicals such as phenolics (especially flavonoids), vitamin C and carotenoids, which contribute to health (Cao et al., 2009). The flowers (inflorescences) and leaves have been widely used in traditional Chinese medicine (TCM) for the treatment of colds, cough, and so on, and thus, loquat has high nutritional and medicinal value and is used in various countries (Zhou et al., 2011). To date, loquat has become very popular and is widely cultivated in many Asian, European, and American countries (Xu et al., 2010; 2011). There are abundant resources of wild and cultivated varieties in China (Qiu and Zhang, 1996).

DNA markers are a category of powerful tools used to identify plant cultivars and species, but there is no strong link between marker information and concrete plant varieties, and these markers have not yet been efficient in the identification of fruit crop varieties ( $\mathrm{Li}$ et al., 2011; Sun et al., 2012). On the basis of these characteristics, Zhao et al. (2011) came up with a novel strategy for the efficient identification of plant individuals relying on a new way of recording DNA fingerprints of genotyped plants, whereby a manual cultivar identification diagram (MCID) can be generated and used as a source of referable information for quick identification of plant and/or seed samples even in the future (Korir et al., 2013). To date, MCID has been successfully used in cultivar identification of many fruit species, including fruiting mei (Li et al., 2011), flowering mei (Wang et al., 2011), grape (Zhao et al., 2011), lemon (Mu et al., 2012), loose-skin mandarin (Leng et al., 2011), pear (Lin et al., 2011), pomegranate (Zhang et al., 2012), sweet orange (Sun et al., 2012), and so on.

EST-SSR (expressed sequence tag-simple sequence repeats) have been applied in genetic studies of fruiting trees, including genetic diversity analysis (Li et al., 2010a), genetic linkage map construction (Chen et al., 2008), comparative mapping (Vendramin et al., 2007), molecular phylogeny and cultivar identification (Nakagawa et al., 2008). Since the EST-SSR markers are derived from transcribed regions of DNA, they are expected to be more conserved and have a higher rate of transferability and polymorphism than genomic SSR markers (Decroocq et al., 2003). Therefore, in view of the utilization of the transferability of EST microsatellite markers across taxa or from related species (Saha et al., 2004; Sim et al., 2009), ESTSSR markers could be an alternative approach for species with few EST sequences distributed in the NCBI (National Center for Biotechnology Information) database.

Compared to other main fruit crops, loquat has not been accorded much attention from geneticists and molecular biologists. Although many kinds of DNA markers have been used in loquat variety identification and genetic diversity (Soriano et al., 2005; Gis- 
bert et al., 2009; Wang et al., 2010; Zhang et al., 2011; Xie et al., 2012), there is no strong link between the marker information and concrete plant varieties, and the markers have not yet made the identification of loquat varieties an efficient, recordable, or easy task. In this study, the majority of 25 EST-derived SSR loci from apple revealed cross-species amplification with alleles of comparable sizes in loquat. As expected, the transferability rate of the markers was high, which suggests that the flanking regions of these SSR loci are sufficiently conserved, and that the markers with polymorphic fingerprints can be used for cultivar identification of loquat. Here, we employed MCID strategy and successfully identified twenty-four cultivars of red-flesh loquat by using seven polymorphic EST-SSR markers. This identification generated a cultivar identification diagram (CID) presenting the polymorphic bands and corresponding primers to be used for the identification of some groups of cultivars. The results of the validation test for the red-flesh loquat CID also confirmed that the MCID method using EST-SSR markers was accurate and reliable and that it can be applied as a scale-like scheme for providing the information needed for separating cultivars as desired. The MCID method can also be used in genetic diversity analysis and genetic improvement in loquat.

\section{MATERIAL AND METHODS}

\section{Plant materials}

Leaf samples of 24 important loquat cultivars (Table 1) were collected from the loquat germplasm collection at the Yangdu Agricultural Research Station, Institute of Horticulture, Zhejiang Academy of Agricultural Sciences, Hangzhou, China. The primers used were synthesized by Shanghai Jierui Biotechnology Company.

Table 1. Summaries of red-fleshed loquat varieties used in this study.

\begin{tabular}{lllc}
\hline Code & Cultivar name & Origin & Fruit characteristic \\
\hline 1 & Dawuxing & Sichuan, China & Red \\
2 & Oohusa & Japan & Red \\
3 & Luoyangqing & Huangyan, Zhejiang, China & Red \\
4 & Algeie & Spain & Red \\
5 & Pelucheis & Spain & Red \\
6 & Ullera & Spain & Red \\
7 & Marc & Spain & Red \\
8 & Touzao & Yuhang, Zhejiang, China & Red \\
9 & Chihong & Yuhang, Zhejiang, China & Red \\
10 & Liufenzhong & Yuhang, Zhejiang, China & Red \\
11 & Baimaowuer & Yuhang, Zhejiang, China & Red \\
12 & Dameiguihongpao & Yuhang, Zhejiang, China & Red \\
13 & Dayeyangdun & Yuhang, Zhejiang, China & Red \\
14 & Jiantoudahongpao & Yuhang, Zhejiang, China & Red \\
15 & Baozhu & Yuhang, Zhejiang, China & Red \\
16 & Jiajiao & Yuhang, Zhejiang, China & Red \\
17 & Erzao & Yuhang, Zhejiang, China & Red \\
18 & Dahongpao & Yuhang, Zhejiang, China & Red \\
19 & Taipinghong & Lishui, Zhejiang, China & Red \\
20 & Taxiahong 1 & Lishui, Zhejiang, China & Red \\
21 & Changhongpipa & Lishui, Zhejiang, China & Red \\
22 & Daguotaipinghong & Lishui, Zhejiang, China & Red \\
23 & Taxiahong 2 & Lishui, Zhejiang, China & \\
24 & Taxiahong & Lishui, Zhejiang, China & \\
\hline
\end{tabular}




\section{DNA extraction}

Fresh leaves of 24 cultivars (Table 1) were collected for DNA extraction and flash frozen in liquid nitrogen, and the powder stored at $-70^{\circ} \mathrm{C}$ until use. Genomic DNA was isolated from leaves according to the modified CTAB method [total volume of $100 \mathrm{~mL}: 2 \mathrm{~g}$ CTAB; 5.84 g EDTA; $1.21 \mathrm{~g}$ Tris; $8.19 \mathrm{~g} \mathrm{NaCl} ; 2 \mathrm{~g}$ polyvinylpyrrolidone (PVP); $2 \% \beta$-mercaptoethanol $(\mathrm{v} / \mathrm{v})]$, and then purified and checked for quality by electrophoresis on a $1 \%$ agarose gel. The concentrations and purity were determined using a Bio-Photometer (Eppendorf). DNA was diluted to $30-60 \mathrm{ng} / \mu \mathrm{L}$ and stored at $-20^{\circ} \mathrm{C}$ until used.

\section{PCR amplification and primer selection}

Twenty-five EST-SSR primer pairs from apple described by Li et al. (2012) were used for PCR amplification. PCR amplification was carried out in a $20-\mu \mathrm{L}$ reaction system, containing $2 \mu \mathrm{L} 30 \mathrm{ng} / \mu \mathrm{L}$ genomic DNA, $0.8 \mu \mathrm{L}(10 \mu \mathrm{moL})$ each primer, $10 \mu \mathrm{L} 2 \mathrm{X}$ Taq mix and 6.4 $\mu \mathrm{L} \mathrm{ddH}_{2} \mathrm{O}$. Amplification reactions were performed in a Huayue Biometra Thermalcycler, using the following program: initial denaturation for $5 \mathrm{~min}$ at $94^{\circ} \mathrm{C}, 38$ cycles of denaturation at $94^{\circ} \mathrm{C}$ for $30 \mathrm{~s}$, corresponding annealing temperature for $30 \mathrm{~s}$ (Table 2), and extension at $72^{\circ} \mathrm{C}$ for $1 \mathrm{~min}$, and a final extension step at $72^{\circ} \mathrm{C}$ for $10 \mathrm{~min}$. The PCR products were checked on a $1.5 \%$ agarose gel first; the primers with corresponding bands were resolved on non-denaturing polyacrylamide gel electrophoresis (PAGE) to check the DNA banding patterns, and the primers that yielded polymorphic bands were used for the loquat cultivar identification step.

\section{Data statistics and cultivar identification}

Only clear unambiguous bands were manually scored from photographic prints of gels for each cultivar. For cultivar identification, the MCID method was employed here (Lin et al., 2011) and cultivars with a specific band in the fingerprint generated from one primer pair could be separated singly, with cultivars sharing the same banding pattern being separated into the same subgroup. On the basis of these criteria, these cultivars were classified into different groups according to the fingerprint amplified by each primer pair. All 24 loquat cultivars were gradually separated from each other as more primers were employed and a final loquat cultivar identification diagram (CID) comprising bands with specific sizes used to separate the cultivars and the related primers that generated the specific bands was constructed following the strategy reported by Wang et al. (2011).

\section{Detection of the workability of loquat CID}

The utilization and workability of the loquat CID was tested according to Zhang et al. (2011). The methods were as follows: two loquat cultivars were randomly selected and used to verify the accuracy of the MCID method. The specific primers that could amplify the polymorphic fragments to be used in separating the cultivars could be easily found on the loquat CID. If the cultivars could be well distinguished as anticipated, this would definitely assure the workability and efficiency of the MCID approach in loquat cultivar identification, and the PCR reaction was established as described above. 


\section{RESULTS}

\section{Transferability of apple EST-SSRs in loquat}

To make the most use of apple EST sequences in developing transferable EST-SSR in loquat, some EST-SSR markers developed from apple were tested in this study for intraspecific transferability in loquat. Apple is genetically close to loquat, and thus, EST-SSRs developed in apple could theoretically have high transferability in loquat. In this study, 25 primer pairs of apple EST-SSR were tested for PCR amplification in loquat cultivars. The results showed that the EST-SSR primers from apple had a relatively high transferability rate of $84 \%$ in loquat, four of the EST-SSR primer pairs failed in producing clear PCR products in loquat, while the other 21 EST-SSR primer pairs generated clear PCR banding patterns, of which 11 pairs amplified polymorphic bands. Those primers with polymorphic bands were used for identification of loquat cultivars.

\section{Cultivar identification}

From the study on the identification of flowering cultivar identification, the MCID strategy can be a preferable choice in the practical utilization of SSR markers in a comp lete and efficient identification of plant cultivars (Wang et al., 2011). For identification of the 24 red-fleshed loquat cultivars, the 21 workable EST-SSR primer pairs from apple were randomly chosen and gradually used to separate the loquat cultivars. After seven primer pairs (Table 2) were utilized, all 24 cultivars used in this study were successfully separated. Of the 7 primer pairs used, primers L 38 and L25 whose PCR patterns are shown in Figure 1, were the first to be used in the identification of all the 24 red-fleshed cultivars. Three polymorphic bands with sizes of 245,227 , and $223 \mathrm{bp}$ were chosen to separate the loquat cultivars, and the presence and absence of these bands could classify the 24 cultivars into five groups as shown in Figure 2. Two cultivars named 'Dawuxing' and 'Erzao' were successfully separated by the presence of 245, 227, 223, and 223 bp, and the two cultivars 'Liufenzhong' and 'Jiantoudahongpao' were clustered into a small group, which were further identified as a single-cultivar group by the 310-bp band amplified by primer L42. Likewise, two other groups of cultivars separated by primer L42 were also gradually separated by several primers marked on the diagram. Finally, all the red-flesh loquat cultivars were successfully separated by the 7 primer pairs shown in Table 2, from which one CID was constructed (Figure 2), making the identification of $\mathrm{f}$ red-flesh loquat cultivars an efficient, referable and easy task. This red-flesh loquat CID could generate referable information that could show the specific primers and polymorphic markers necessary to separate and identify the cultivars.

\section{Validation and workability of the red-fleshed loquat CID}

To confirm the workability and efficiency of the CID generated above, scientific validation was need, for which 12 loquat cultivars in different groups including 'Oohusa' and 'Liufenzhong', 'Ullera' and 'Jiantoudahongpao', 'Baimaowuer' and 'Changhongpipa', 'Dameiguihongpao' and 'Baozhu', 'Pelucheis' and 'Marc', and 'Chihong' and 'Jiajiao', were randomly chosen. From the location of these cultivars in the CID (Figure 2), it was easy to find 
that the primers L38, L42, L25, and L16 were definitely those to be used in separating each of the cultivar pairs. Clearly, the verification of the PCR results of these groups of cultivars using the corresponding primers were the same as those anticipated (Figure 3 ). The first three groups above could be validated with the primer pair L38 (Figure 3A, B, C). The forth group of 'Dameiguihongpao' and 'Baozhu' could be separated by primer pair L42 (Figure 3D). The fifth and sixth groups, i.e., 'Pelucheis' and 'Marc' and 'Chihong' and 'Jiajiao', were separated by L25 (Figure 3E) and L16 (Figure 3F), respectively. Using this procedure, each two cultivars selected were successfully identified with the corresponding combination of primers. Therefore, this detection proved the availability, workability and efficiency of the MCID method in red-fleshed loquat cultivar identification, by using the EST-SSR markers in this study.

\begin{tabular}{|c|c|c|c|c|}
\hline SSR code & Primer sequence $\left(5^{\prime} \rightarrow 3^{\prime}\right) *$ & $\operatorname{Tm}\left({ }^{\circ} \mathrm{C}\right)$ & No. of alleles & Size of alleles (bp) \\
\hline L6 & $\begin{array}{l}\text { F: CAAAGATCAAGTAACGGGACAA } \\
\text { R: CACATACCACACTTTGCCACA }\end{array}$ & 57.6 & 1 & 198 \\
\hline L16 & $\begin{array}{l}\text { F: ATCCATGGCCAAATGAAAAC } \\
\text { R: TGAGATTAAGACCGGGCATC }\end{array}$ & 55.9 & 1 & 172 \\
\hline L20 & $\begin{array}{l}\text { F: CGCTTTTCTTTGTCGCTTTC } \\
\text { R: TGGTTTTCATGGAGGAGGAG }\end{array}$ & 55.5 & 1 & 198 \\
\hline L25 & $\begin{array}{l}\text { F: CTACCTCACCGGGAAGATGA } \\
\text { R:GAGACACGTACATGATAAGACCA }\end{array}$ & 56.5 & 2 & 210,160 \\
\hline L38 & $\begin{array}{l}\text { F: TTCCAAATTCCAAAGCCTTC } \\
\text { R: CGAAAAAGTGGACCGAACAC }\end{array}$ & 57 & 3 & $245,227,223$ \\
\hline L42 & $\begin{array}{l}\text { F:TCACAGCCTCGAAAGACAGA } \\
\text { R: ATCACCAGCAGGGAAGAATG }\end{array}$ & 58 & 1 & 310 \\
\hline L48 & $\begin{array}{l}\text { F: CATTTGATAAACGTACCCAGCA } \\
\text { R: CGCCGCTACATGAGAAAACT }\end{array}$ & 59 & 3 & $320,190,180$ \\
\hline
\end{tabular}

*Every two primers belong to one pair. $\mathrm{F}$ is the forward and $\mathrm{R}$ is the reverse primer.

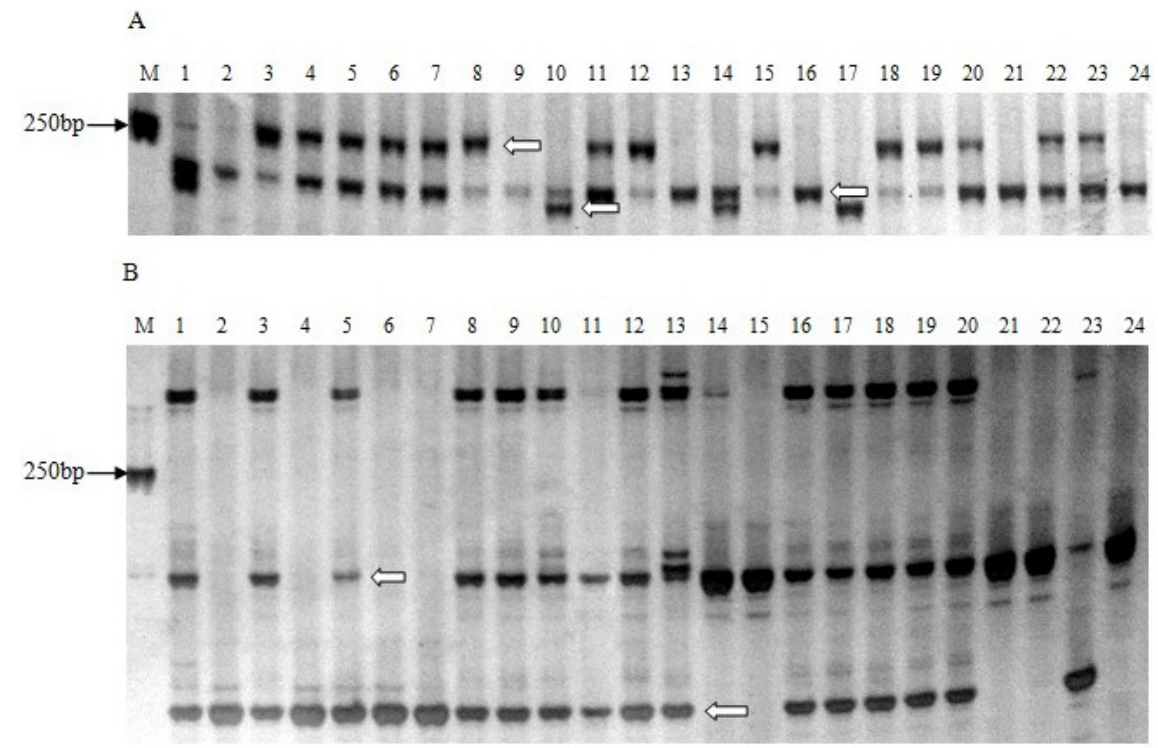

Figure 1. DNA banding patterns of 24 red-flesh loquat cultivars amplified by primers L38 and L25, respectively. Lane $M=$ DL2000 marker; lanes 1-24 = accession numbers of loquat cultivars listed in Table 1. A. Amplified result of L38; B. amplified result of primer L25. 


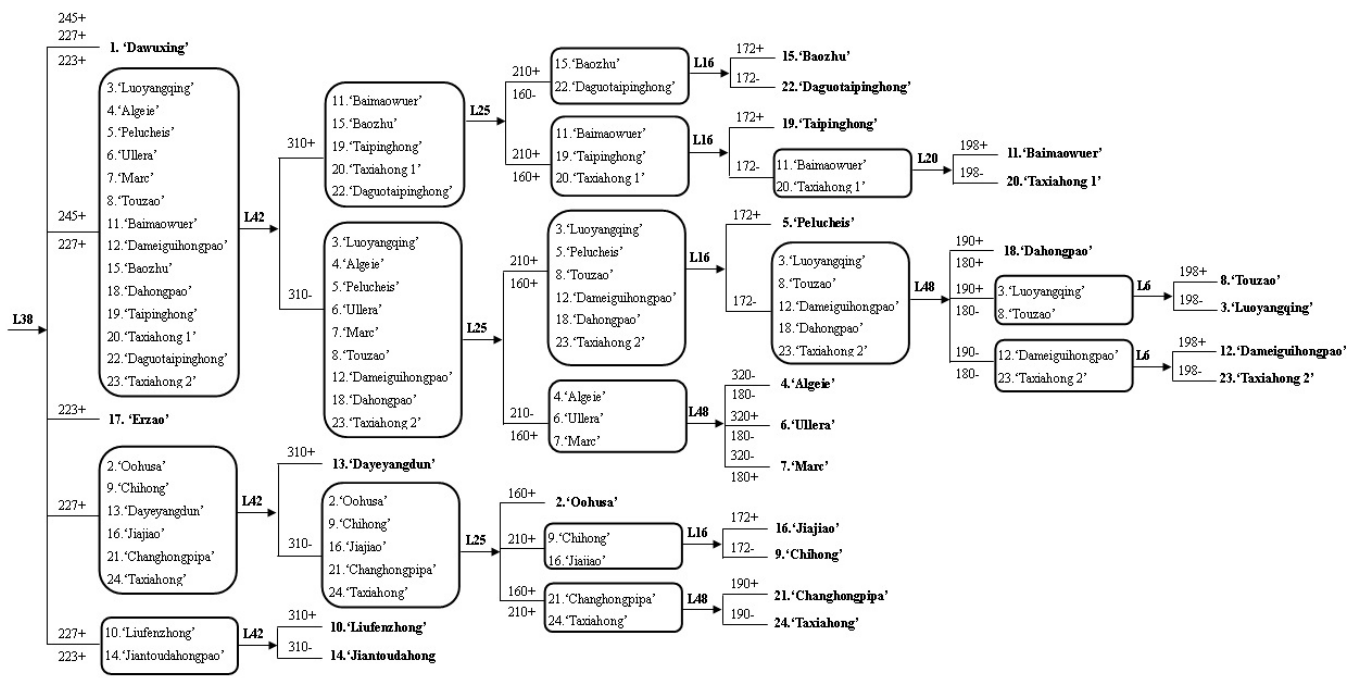

Figure 2. Identification results of red-fleshed loquat cultivars used by 7 pairs of EST-SSR primers and corresponding polymorphic fingerprints. All the numbers marked in this CID indicated different size fingerprints, and the unit is "bp"; $(+)=$ bands in different size were existed; $(-)=$ bands in different size were absent.

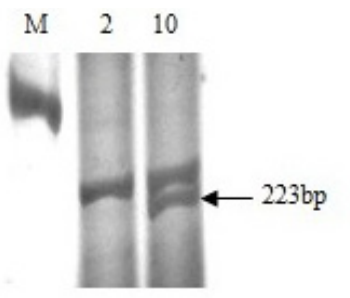

A

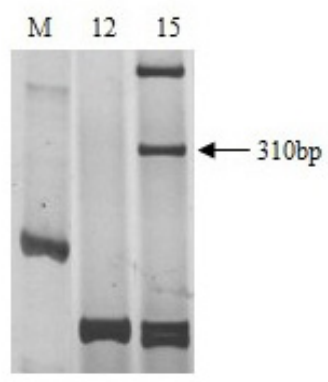

$\mathrm{D}$

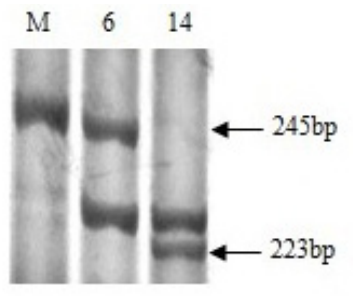

B

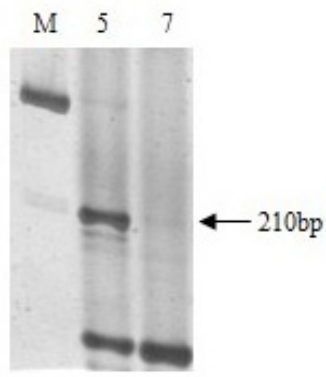

$\mathrm{E}$

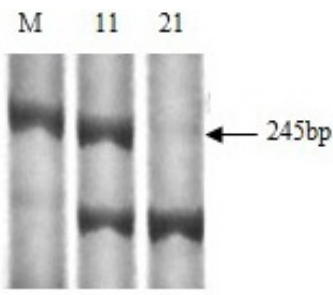

C

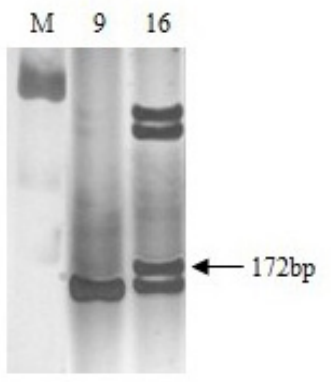

F

Figure 3. Verification results of 12 cultivars randomly selected amplified by the corresponding primers in loquat CID. Lane $M=$ DL2000 plus marker; Numbers = accession numbers and names of these cultivars used as listed in Table 1. A. B. C. Amplified by EST-SSR primer L38; D. amplified by L42; E. amplified by L25; F. amplified by L16. 


\section{DISCUSSION}

Plant cultivar/variety identification is a critical field and as more and more varieties/ cultivars are developed, the need to properly identify and categorize them increases. There is a growing need to refine the methods of recording, analyzing, presenting, storing, and retrieving information on morphological descriptors of existing and emerging plant genotypes (Korir et al., 2013). Traditional approaches to plant cultivar identification, such as morphological, paleontological, cytological, isozyme, etc., have proven to be limited by difficult operability, low stability and reproducibility, susceptibility to environmental influences, and the need for extensive observation in mature plants. DNA-based fingerprinting markers overcome these limitations and can provide a powerful tool for proper characterization of cultivars ( $\mathrm{Li}$ et al., 2011). In recent years, various DNA molecular markers have been developed and used for studies on genetic diversity, fingerprinting patterns and origins of the cultivars (Cheng and Huang, 2009; D’Onofrio et al., 2009; Lu et al., 2009; Melgarejo et al., 2009; Li et al., 2010b).

Microsatellites have been used as markers in different plant species, because of their variability, ease of use, accessibility of detection and reproducibility (Fu et al., 2011), which are hypervariable single-locus markers that have been previously shown to be highly informative in the identification of varieties/cultivars. In general, SSR are derived from either genomic DNA or cDNA sequences (Chen et al., 2006). However, the development of SSR from genomic DNA is still expensive and time consuming, since it involves the construction of genomic DNA libraries, probe hybridization, cloning and sequencing (Roder et al., 1998). With the growth of EST databases, an alternative strategy to develop SSR markers is to search for EST-SSRs from ESTs deposited in public databases through data mining, which has been widely carried out for many plant species (Celton et al., 2009; Bassil and Postman, 2010). Compared to genomic SSR, the advantage of EST-SSRs is that they are more conserved, and may be a marker linked to candidate genes of plant development or dominant resistance genes.

Red-flesh loquat originates from China, which has a long history and is widely cultivated in many countries. It has seen increased cultivation with growing market demand around the world. To the best of our knowledge, not many loquat ESTs have been submitted to NCBI, and hardly any EST-SSRs have been developed. Considering the high degree of transferability of SSRs (Soriano et al., 2005; Gisbert et al., 2009), it is important to develop more loquat ESTSSR markers by screening transferable EST-SSRs from other plants. According to current research, several reports have focused on SSR development and their transferability across the Rosaceae (Vendramin et al., 2007; Gasic et al., 2009).

Loquat is genetically related to apple (Malus domestica), and they both belong to the same subgenus Maloideae. Because of the availability of a large number of apple ESTs, the development of loquat EST-SSRs from apple can be preferable based on the reports that EST-SSR markers could be transferable across species in the genus Malus. This study was initiated to investigate the transferability of apple EST-SSR markers to red-flesh loquat. In this study, the majority of 25 EST-derived SSR loci from apple revealed cross-species amplification with alleles of comparable sizes in red-flesh loquat. As expected, the transferability rate of the markers was high, reaching $84 \%$, which suggests that the flanking regions of these SSR loci are sufficiently conserved and can be used for comparative analyses of genetic diversity, cultivar identification and so on. Compared to other fruit crops, the transferability rate of apple EST-SSR markers across species was much higher than that other Rosaceae species, such 
as apricot, pear, peach, strawberry (Gasic et al., 2009). Our results indicate a relatively high level of transferability between apple and loquat, and that EST-SSR marker development from apple has great application value. The transferable SSR loci identified in the present study can be of use in genetic analysis and cultivar identification of red-flesh loquat.

On the basis of the effective EST-SSR markers obtained from apple, MCID strategy was further employed gradually distinguishing the red-fleshed loquat cultivars, according to the polymorphic bands amplified by EST-SSR markers. Finally, seven EST-SSR markers were used to successfully identify the 24 red-flesh loquat cultivars. The cultivar identification diagram for them was then generated, and the information on the polymorphic markers was used to separate the loquat cultivars on it, which is quite different from the unweighted pair-group method (UPGMA) for clustering, which is computer-based (Gisbert et al., 2009). Once new red-flesh loquat cultivars are released, the set of 7 EST-SSR primers could be used to amplify the DNA of the new cultivars, and the PCR bands appearing on the PAGE gel can let us know where to position the new cultivars in the CID already constructed. But, if all primers used originally, such as the 7 in this study, cannot provide clear separation of the new cultivars, additional EST-SSR primers need to be screened and used to separate these new cultivars. With the identification information of the new cultivars, a larger red-flesh loquat CID can be developed. That is to say, less work is needed to distinguish one or several new cultivars. It is recommended that this study will be widened to include more varieties, landraces and other red-flesh genotypes as well as a bigger array of EST-SSR markers to be used. It is also noted that the validation results of the CID here can also confirm the practical importance of loquat cultivar identification using MCID through EST-SSR markers, which is workability and accuracy as anticipated.

In summary, our results suggest that EST-SSR markers developed from apple are useful and have great application value. The transferable SSR loci identified in the present study can be further used in genetic analysis and cultivar identification of more loquat cultivars and even other Rosaceae species. MCID based on EST-SSR markers will be essential in granting protection to all the new loquat varieties through DUS (distinctness, uniformity, and stability) testing ( $\mathrm{Lu}$ et al., 2009).

\section{ACKNOWLEDGMENTS}

Research supported by China Postdoctoral Science Foundation (\#2013M531482); Key Project for New Agricultural Cultivar Breeding in Zhe $\neg$ jiang Province, China (\#2012C129045); National Science Foundation for Distinguished Young Scholars of China (\#31101530); the Natural Science Foundation of Zhejiang Province, China (\#Y3110609), Important Item for Scientific and Technical Innovation Team of Zhejiang Province, China (\#2009R50033).

\section{REFERENCES}

Bassil N and Postman JD (2010). Identification of European and Asian pears using EST-SSRs from Pyrus. Genet. Resour. Crop Evol. 57: 357-370.

Cao SF, Zheng YH, Yang ZF, Wang KT, et al. (2009). Effect of methyl jasmonate on quality and antioxidant activity of postharvest loquat fruit. J. Sci. Food Agric. 89: 2064-2070.

Celton JM, Tustin DS, Chagné D and Gardiner SE (2009). Construction of a dense genetic linkage map for apple rootstocks using SSRs developed from Malus ESTs and Pyrus genomic sequences. Tree Genet. Genomes 5: 93-107. 
Chen C, Zhou P, Choi YA, Huang S, et al. (2006). Mining and characterizing microsatellites from citrus ESTs. Theor. Appl. Genet. 112: 1248-1257.

Chen C, Bowman KD, Choi YA, Dang PM, et al. (2008). EST-SSR genetic maps for Citrus sinensis and Poncirus trifoliate. Tree Genet. Genomes 4: 1-10.

Cheng ZP and Huang HW (2009). SSR fingerprinting Chinese peach cultivars and landraces (Prunus persica) and analysis of their genetic relationships. Sci. Hortic. 120: 188-193.

D’onofrio C, Lorenzis G, De Giordani T, Natali L, et al. (2009). Retrotransposon-based molecular markers in grapevine species and cultivars identification and phylogenetic analysis. Acta Hortic. 827: 45-52.

Decroocq V, Fave MG, Hagen L, Bordenave L, et al. (2003). Development and transferability of apricot and grape EST microsatellite markers across taxa. Theor. Appl. Genet. 106: 912-922.

Fu J, Peng ZJ, Cai XD and Guo WW (2011). Regeneration and molecular characterization of interspecific somatic hybrids between Satsuma mandarin and two seedy sweet oranges for scion improvement. Plant Breed. 130: 287-290.

Fu X, Kong W, Peng G, Zhou J, et al. (2012). Plastid structure and carotenogenic gene expression in red- and white-fleshed loquat (Eriobotrya japonica) fruits. J. Exp. Bot. 63: 341-354.

Gasic K, Han Y, Kertbundit S, Shulaev V, et al. (2009). Characteristics and transferability of new apple EST-derived SSRs to other Rosaceae species. Mol. Breed. 23: 397-411.

Gisbert AD, Martínez-Calvo J, Llácer G, Badenes ML, et al. (2009). Development of two loquat [Eriobotrya japonica (Thunb.) Lindl.] linkage maps based on AFLPs and SSR markers from different Rosaceae species. Mol. Breed. 23: 523-538.

Korir NK, Han J, Shangguan L, Wang C, et al. (2013). Plant variety and cultivar identification: advances and prospects. Crit. Rev. Biotechnol. 33: 111-125.

Leng XP, Li HR, Zhong GY, Song CN, et al. (2011). Employment of a new strategy for identification of loose-skin mandarin (Citrus reticulata Blanco) cultivars using RAPD markers. Rom. Biotech. Lett. 17: 7073-7083.

Li HR, Wang K, Sun X, Zhang CH, et al. (2012). Development of EST-derived SSR markers and their applications in apple genetic diversity analysis. Jiangsu J. Agric. Sci. 28: 158-165.

Li X, Shangguan L, Song C, Wang C, et al. (2010a). Analysis of expressed sequence tags from Prunus mume flower and fruit and development of simple sequence repeat markers. BMC Genet. 11: 66.

Li X, Wang Y, Wang B, Wang C, et al. (2010b). Genetic relationships between fruiting and flowering mei (Prunus mume) cultivars using SNP markers. J. Hortic. Sci. Biotech. 85: 329-334.

Li XY, Wang C, Yang G, Li XD, et al. (2011). Employment of a new strategy for identification of Prunus mume cultivars using random amplified polymorphic deoxyribonucleic acid (RAPD) markers. Afr. J. Plant Sci. 5: 500-509.

Lin J, Wang XC, Chang YH and Fang JG (2011). Development of a novel and efficient strategy for practical identification of Pyrus spp (Rosaceae) cultivars using RAPD fingerprints. Genet. Mol. Res. 10: 932-942.

Lu X, Liu L, Gong Y, Zhao L, et al. (2009). Cultivar identification and genetic diversity analysis of broccoli and its related species with RAPD and ISSR markers. Sci. Hortic. 122: 645-648.

Melgarejo P, Martínez J, Hernández F, Martínez R, et al. (2009). Cultivar identification using 18S-28S rDNA intergenic spacer-RFLP in pomegranate (Punica granatum L.). Sci. Hortic. 120: 500-503.

Mu Q, Sun X, Zhong GY, Wang XC, et al. (2012). Employment of a new strategy for identification of lemon (Citrus limon L.) cultivars using RAPD markers. Afr. J. Agr. Res. 7: 2075-2082.

Nakagawa T, Nakatsuka A, Yano K, Yasugahira S, et al. (2008). Expressed sequence tags from persimmon at different developmental stages. Plant Cell Rep. 27: 931-938.

Qiu WL and Zhang HZ (1996). China Fruit Records - Longan and Loquat (in Chinese). (Beijing, ed.). China Forestry Press.

Roder MS, Korzun V, Wendehake K, Plaschke J, et al. (1998). A microsatellite map of wheat. Genetics 149: 2007-2023.

Saha MC, Mian MA, Eujayl I, Zwonitzer JC, et al. (2004). Tall fescue EST-SSR markers with transferability across several grass species. Theor. Appl. Genet. 109: 783-791.

Sim SC, Yu JK, Jo YK, Sorrells ME, et al. (2009). Transferability of cereal EST-SSR markers to ryegrass. Genome 52: 431-437.

Soriano JM, Romero C, Vilanova S, Llacer G, et al. (2005). Genetic diversity of loquat germplasm [Eriobotrya japonica (Thunb) Lindl] assessed by SSR markers. Genome 48: 108-114.

Sun X, Mu Q, Jiang D, Wang C, et al. (2012). A new strategy employed for identification of sweet orange cultivars with RAPD markers. Genet. Mol. Res. 11: 2071-2080.

Vendramin E, Dettori MT, Giovinazzi J, Micali S, et al. (2007). A set of EST-SSRs isolated from peach fruit transcriptome and their transportability across Prunus species. Mol. Ecol. Notes 7: 307-310.

Wang Y, Fu Y, Yang Q, Luo N, et al. (2010). Genetic diversity of Eriobotrya analyzed by ISSR markers. Sci. Silvae Sin. 46: 49-57. 
Wang YJ, Li XY, Han J, Fang WM, et al. (2011). Analysis of genetic relationships and identification of flowering-mei cultivars using EST-SSR markers developed from apricot and fruiting-mei. Sci. Hortic. 132: 12-17.

Xie LX, Xu JH, Zhang LJ, Zhang XY, et al. (2012). Genetic relationship analysis of 24 white-fleshed loquat (Eriobotrya japonica) germplasm by ISSR. Fujian J. Agric. Sci. 27: 261-266.

Xu HX, Chen JW and Xie M (2010). Effect of different light transmittance paper bags on fruit quality and antioxidant capacity in loquat. J. Sci. Food Agric. 90: 1783-1788.

Xu HX and Chen JW (2011). Commercial quality, major bioactive compound content and antioxidant capacity of 12 cultivars of loquat (Eriobotrya japonica Lindl.) fruits. J. Sci. Food Agric. 91: 1057-1063.

Zhang X, Qian J, Wang H, Yuan W, et al. (2011). A novel strategy employed in identification of 25 important loquat cultivars using RAPD marker. Caryologia 64: 265-271.

Zhang YP, Tan HH, Cao SY, Wang XC, et al. (2012). A novel strategy for identification of 47 pomegranate (Punica granatum) cultivars using RAPD markers. Genet. Mol. Res. 11: 3032-3041.

Zhao MZ, Zhang YP, Wu WM, Wang C, et al. (2011). A new strategy for complete identification of 69 grapevine cultivars using random amplified polymorphic DNA (RAPD) markers. Afr. J. Plant Sci. 5: 273-280.

Zhou C, Sun C, Chen K and Li X (2011). Flavonoids, phenolics, and antioxidant capacity in the flower of Eriobotrya japonica Lindl. Int. J. Mol. Sci. 12: 2935-2945. 